\title{
SPOLM2O19
}

XIX SIMPÓSIO DE PESQUISA OPERACIONAL E LOGÍSTICA DA MARINHA RIO DE JANEIRO, RJ, BRASIL - 06 A 08 DE NOVEMBRO DE 2019

\section{A INTERFERÊNCIA DE ÂNGULOS DE GIRO NO TEMPO DE EVACUAÇÃO DE MULTIDÕES EM SITUAÇÃO DE NORMALIDADE E DE PÂNICO}

\author{
Manuela Marques Lalane Nappi \\ Universidade Federal de Santa Catarina \\ Campus Reitor João David Ferreira Lima, s/n - Trindade, Florianópolis - SC, 88040-900 \\ lalppi.ms@gmail.com \\ Maria Luiza Tremel de Faria Lima \\ Universidade Federal de Santa Catarina \\ Campus Reitor João David Ferreira Lima, s/n - Trindade, Florianópolis - SC, 88040-900 \\ marialuizatfaria@hotmail.com \\ João Carlos Souza \\ Universidade Federal de Santa Catarina \\ Campus Reitor João David Ferreira Lima, s/n - Trindade, Florianópolis - SC, 88040-900 \\ joao.carlos@ufsc.br
}

\section{RESUMO}

A segurança das pessoas em locais de reunião de grande público apresenta implicações significativas, entre outras coisas, para o planejamento urbano, gerenciamento de eventos, projeto de edifícios, segurança contra incêndio e serviço de resgate. Comportamentos complexos de multidões de pedestres podem ser críticos sob condições de emergência e alta densidade, como manobras de virada, interseção e fusão. O objetivo deste trabalho foi verificar a influência de diferentes ângulos de giro no tempo de escape de uma multidão de um ambiente fechado a partir de simulações computacionais baseadas no modelo de força social. Em todos os cenários analisados buscou-se verificar o fluxo de escape de 250 pessoas de uma sala de $1000 \mathrm{~m}^{2}$ por um ou mais corredores que exigiam diferentes ângulos de giro ao serem percorridos. Todos os cenários foram simulados para duas velocidades diferentes: normal de caminhada dentro de uma multidão e de nervosismo. Os resultados encontrados apontam que os ganhos relacionados ao aumento da eficácia de uma evacuação podem chegar a 33,36\% quando considerados diferentes ângulos de giro e a 17,11\% quando consideradas diferentes quantidades e posicionamento das saídas de emergência.

Palavra-chave: Arquitetura; Evacuação Emergencial; Ângulos de Giro; Simulação computacional; Modelo de Força Social.

\begin{abstract}
The safety of people in general meeting places has significant implications, for urban planning, event management, building design, fire safety and rescue services, among others. Complex behaviors of pedestrian crowds can be critical under emergency and high-density conditions, such as turning points intersection and fusion maneuvers. The objective of this work was to verify the influence of different turning angles on the escape time of a crowd in a closed environment, based on computer simulations applying the social force model. In the
\end{abstract}


scenarios analyzed, the aim was to verify the scape flow of 250 people from a $1000 \mathrm{~m}^{2}$ room via one or more corridors that required different angles of rotation when traveling. All scenarios were simulated for two different speeds: normal walking within a crowd and nervous (fast-paced walking). The results indicate that the gains related to the increase of the effectiveness of an evacuation can reach $33.36 \%$ when considering different angles of turn, and $17.11 \%$ when considering different quantities and positioning of emergency exits.

Keywords: Architecture; Emergency evacuation; Turning angles; Computer simulation; Social Force Model.

\section{Como Citar:}

NAPPI, Manuela Marques Lalane; LIMA, Maria Luiza Tremel de Faria; SOUZA, João Carlos. A Interferência de Ângulos de Giro no Tempo de Evacuação de Multidões em Situação de Normalidade e de Pânico. In: SIMPÓSIO DE PESQUISA OPERACIONAL E LOGÍSTICA DA MARINHA, 19., 2019, Rio de Janeiro, RJ. Anais [...]. Rio de Janeiro: Centro de Análises de Sistemas Navais, 2019.

\section{INTRODUÇÃO}

A segurança das pessoas em locais de reunião de grande público tem sido reconhecida como uma área de pesquisa relevante e em rápido crescimento. Ela apresenta implicações significativas para o planejamento urbano, gerenciamento de eventos, projeto de edifícios, segurança contra incêndio, serviço de resgate, etc. [1]. Sendo assim, compreender o comportamento de multidões durante um deslocamento coletivo pode ser considerado o cerne da engenharia de tráfego de pedestres [2, 3].

Comportamentos complexos de multidões de pedestres podem ser críticos sob condições de emergência e alta densidade, como manobras de virada, interseção e fusão. Além disso, a escassez de dados humanos sobre pânico traz dificuldades e desafios à modelagem da dinâmica de multidões, especialmente quando se trata de situações emergenciais [4]. O pânico pode ser aqui entendido como uma situação em que, devido à alta densidade de uma multidão e ao pouco tempo para a efetiva evacuação, os indivíduos apresentam visão e informação limitadas. O que resulta em competição física e comportamento agressivo [2, 3, 5, 6]. Utilizando-se de dados coletados em experimentos com formigas, Dias et al. [4] avaliaram o impacto de giros, cruzamentos e fusão de configurações em situação de pânico coletivo, tendo identificado a tendência de que, com o aumento do ângulo, a velocidade média de escape diminui, especialmente em altas densidades e sob condições de pânico.

Este trabalho teve como objetivo verificar a influência de diferentes ângulos de giro no tempo de escape de uma multidão de um ambiente fechado a partir de simulações computacionais baseadas no modelo de força social. O software utilizado para as simulações foi o PTV Viswalk, que permite a simulação de pedestres realisticamente, tornando possível a análise de fluxos de pedestres dentro e fora de edifícios [7]. Foram analisados 3 conjuntos de cenários, sendo que o primeiro deles buscou replicar o estudo desenvolvido por Dias et al. [4].

Em todos os cenários analisados buscou-se verificar o fluxo de escape de 250 pessoas de uma sala de $1000 \mathrm{~m}^{2}$ por um ou mais corredores que exigiam diferentes ângulos de giro ao serem percorridos $\left(0^{\circ}, 30^{\circ}, 45^{\circ}, 60^{\circ}\right.$ e $\left.90^{\circ}\right)$. O primeiro conjunto de cenários avaliou o escape por um único corredor com largura de 4,42 m; o segundo e o terceiro conjunto se distinguiram por apresentarem dois corredores, com 2,21 m de largura cada. No segundo conjunto de cenário, os corredores foram dispostos em paredes opostas e, no terceiro, foram 
dispostos na mesma lateral. Todos os cenários foram simulados para duas velocidades diferentes: normal de caminhada dentro de uma multidão $(1,32 \mathrm{~m} / \mathrm{s})$ e de nervosismo $(2,5 \mathrm{~m} / \mathrm{s})$.

Os resultados obtidos com o primeiro conjunto de cenários mostraram-se muito semelhantes àqueles obtidos por Dias et al. [4] para formigas em pânico. Além disso, evidenciaram que o aumento do ângulo de giro repercute diretamente no fluxo de saída (pessoas/s). Os cenários com dois corredores apresentaram-se mais eficazes, para ângulos de giro de $60^{\circ}$ e $90^{\circ}$, quando comparados aos cenários com um único corredor.

1.

\section{MULTIDÕES}

ÂNGULOS DE GIRO E O TEMPO DE ESCAPE DE

Estudos realizados por pesquisadores japoneses [8] demonstraram experimentalmente que a velocidade de caminhada de uma multidão se reduz quando as pessoas realizam manobras de giro. A função de giro, segundo os autores, está relacionada à inércia dos pedestres, uma vez que suas velocidades de caminhada diminuem cada vez que eles se viram. Seus estudos corroboram os achados de pesquisadores franceses [9] que concluíram que o aumento no ângulo de desvio de uma direção é acompanhado pela queda da velocidade média do corpo, ou seja, a velocidade é menor quando se caminha por caminhos curvos do que quando se faz trajetos retos sob condições normais. A principal razão disso, conforme apontado pelos autores, é o desequilíbrio do corpo durante a função de giro.

Alguns acidentes ocorridos em eventos que reúnem multidões ajudam a reforçar a importância de se considerar a presença de ângulos de giro no projeto de edifícios ou de locais que reúnam grande público. Um acidente ocorrido em Kobe, no Japão, em julho de 2001, por exemplo, resultou no atropelamento e esmagamento de pessoas e teve como uma das principais causas a curva existente no viaduto de pedestres de Akashi [5]. Em outro acidente, ocorrido em julho de 2010, na Love Parede (Duisburg, Alemanha) a debandada e esmagamento teria ocorrido devido à existência de percursos inclinados e à fusão de caminhos $[10,11]$.

A redução na velocidade dos pedestres, seja por respostas psicológicas ou físicas, pode criar gargalos quando se trata de uma multidão percorrendo caminhos angulares [4]. Sendo que curvas muito acentuadas reduzem a capacidade de um corredor por fazerem com que os pedestres se locomovam nas pistas mais internas [12]. Diante do que foi exposto, evidencia-se a necessidade de considerar as implicações do fator de giro em situações onde não seja possível contar com corredores ou caminhos retos devido a limitações de espaço, considerações estéticas, etc. [4]. O que também permitiria descrever o comportamento dos pedestres em torno de uma saída de forma mais precisa e realista [8].

2.

\section{MODELAGENS INSPIRADA EM FORMIGAS}

Diversos estudos sugerem que o comportamento coletivo de humanos e formigas durante uma fuga em pânico pode ser bastante semelhante [4, 13-16]. Esses estudos justificariam a viabilidade do uso de dados empíricos coletados com esses organismos não humanos. Nishinari et al. [15], por exemplo, verificaram que formigas seguem outras formigas em função de traços de ferormônios, enquanto pessoas tentam seguir umas às outras, durante as evacuações, com o objetivo de escaparem em segurança e de maneira eficaz.

Shiwakoti et al. [6] testaram a capacidade de um modelo único em descrever com precisão o comportamento de humanos e formigas argentinas (Linepithema humile). Tratamse de duas espécies que, segundo os autores, se diferem na maneira e na velocidade de locomoção bem como na percepção e comunicação química, visual e auditiva e na natureza de sua organização social. Com base nos estudos de um pesquisador do Reino Unido [17], 
Shiwakoti et al. [6] procederam ao reescalonamento de parâmetros a partir de uma relação alométrica usual para o efeito da massa corporal em outras variáveis. Fundamentados na velocidade máxima alcançada por humanos e formigas em curtas distâncias, efetuados os cálculos necessários, assumiram que quaisquer parâmetros que não pudessem ser medidos em suas comparações seguiriam a analogia de serem 1265 vezes maiores para humanos.

Conforme citado anteriormente, Dias et al. [4] realizaram experimentos a fim de testar a influência do ângulo de giro no tempo de escape de cerca de 250 formigas em pânico. Foram considerados quatro ângulos de giro $\left(30^{\circ}, 45^{\circ}, 60^{\circ}\right.$ e $\left.90^{\circ}\right)$ além de um corredor reto, ou seja, giro de $0^{\circ}$. Os autores efetuaram 25 repetições para cada ângulo de giro e registraram o tempo de fuga de 100 formigas, tendo ignorado as 10 primeiras a fim de descartar os atrasos iniciais. Ao compararem corredores retos com corredores angulares, concluíram que ângulos superiores a $45^{\circ}$ diminuem de maneira significativa os tempos de escape em situações de alta densidade. Segundo os autores, esses resultados devem ser verificados considerando-se mais parâmetros, como largura e comprimento dos corredores, diferentes níveis de densidade, etc. De qualquer forma, entendem que o impacto do ângulo de giro no escape de uma multidão não deve ser negligenciado. Dias et al. [4] destacam que o fluxo de saída (formigas / segundo) não diminui continuamente com o aumento do ângulo de giro, especialmente nos ângulos de 60 e 90 graus. Os autores acreditam que alguns ângulos de giro, como o de $60^{\circ}$, possam favorecer os padrões morfológicos e de locomoção do corpo das formigas.

3.

\section{MODELAGENS INSPIRADA EM FORMIGAS}

Neste trabalho foram examinados 15 diferentes projetos de saída de um ambiente fechado, com $1000 \mathrm{~m}^{2}$ cada. Como pode ser observado na Figura 1, os projetos foram divididos em 3 conjuntos: i) com um único corredor de 4,42 m de largura; ii) com dois corredores dispostos em paredes opostas, tendo 2,21 m de largura cada um deles; iii) com dois corredores, também com 2,21 m de largura cada, dispostos na mesma lateral.

Figura 1 - Projetos analisados: (i) com um corredor; (ii) com dois corredores em laterais opostas; e (iii) com dois corredores na mesma lateral

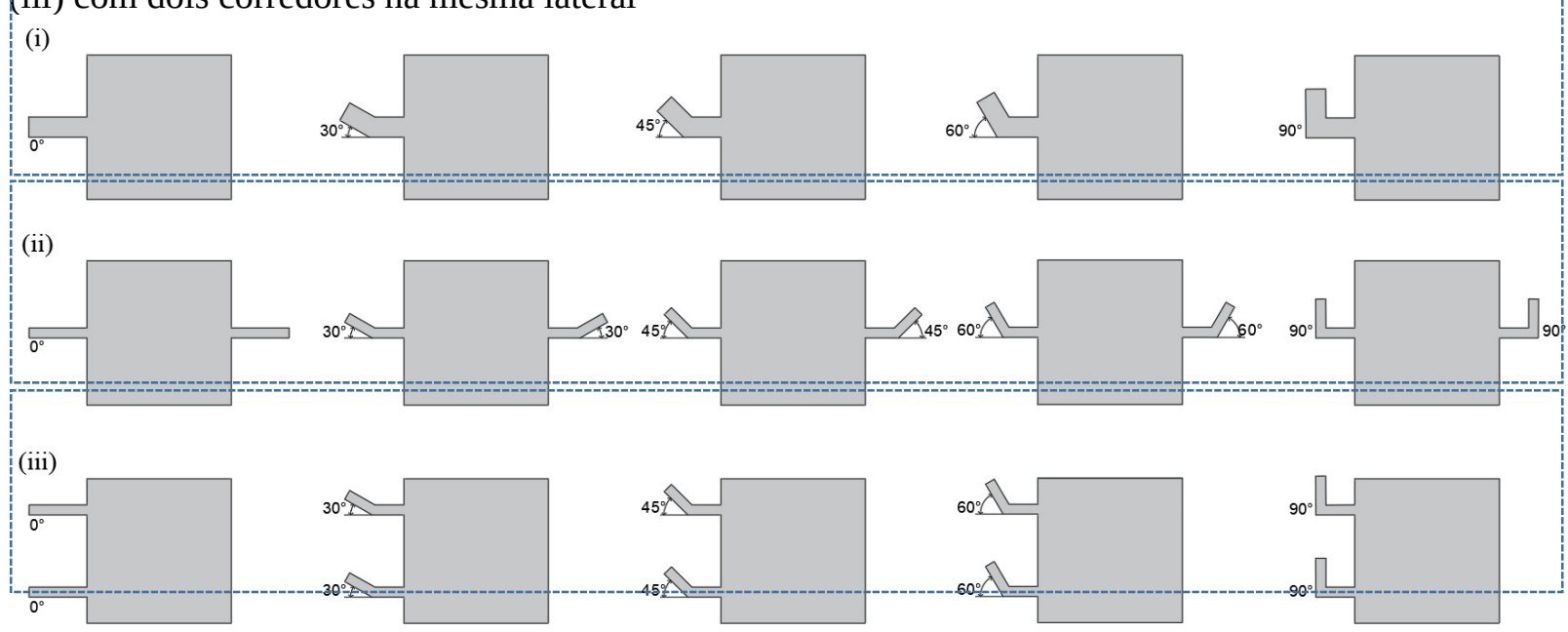

Fonte: Elaborada pelos autores.

Cada um dos projetos foi analisado para duas diferentes velocidades desejadas (Vd): $1,32 \mathrm{~m} / \mathrm{s}$, que corresponde a uma velocidade normal de caminhada dentro de uma multidão [18] e 2,5 m/s, que corresponde à velocidade de caminhada de pessoas com pressa (maior que $1,5 \mathrm{~m} / \mathrm{s}$ ), introduzindo o parâmetro denominado nervosismo às simulações [19]. Considerando-se as duas velocidades imputadas, foram simulados 30 cenários, cada um deles 
com 250 pessoas. Número que buscou repetir as simulações de Dias et al [4], que também serviram de parâmetro para o dimensionamento arquitetônico dos cenários. Para tanto, utilizou-se do reescalonamento de parâmetros proposto por Shiwakoti et al. [6], multiplicando-se as dimensões adotadas por Dias et al. [4] por 1265 (Figura 2).

Figura 2 - Dimensões adotadas, sendo $\mathrm{d}=12,64 \mathrm{~m}$
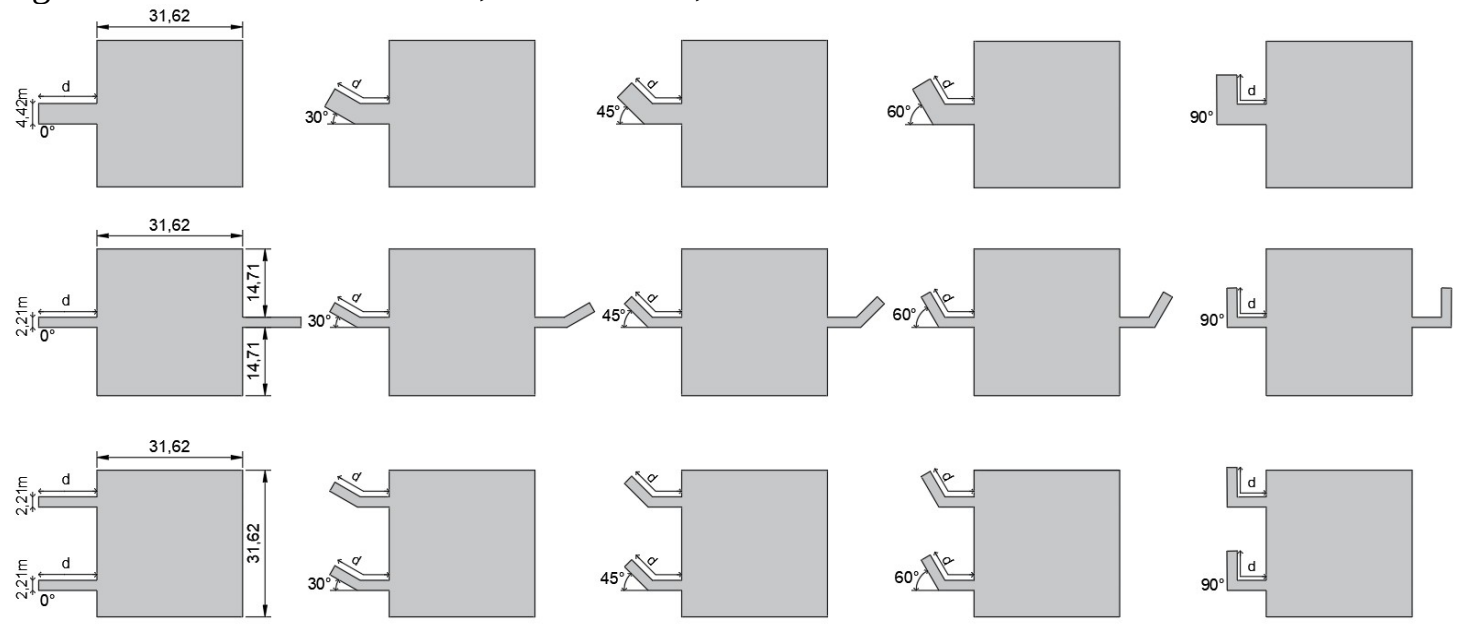

Fonte: Elaborada pelos autores.

O software utilizado para as simulações foi o PTV Vissim, em seu módulo PTV Viswalk, que permite a utilização da abordagem de Força Social. O PTV Viswalk permite que os pedestres andem independentemente do seu destino, ou seja, não há um modelo de rede pré-definido para as suas trajetórias. O software adota um modelo microscópico e assume como padrão o caminho mais curto entre origem e destino de um pedestre, o que foi mantido neste estudo. Destaca-se que a escolha pelo modelo de simulação de Força Social está fundamentada na análise de diversos trabalhos [6, 20-22] bem como na ampla aceitação desse modelo no meio acadêmico [23], já que ele é capaz de modelar diversos comportamentos coletivos, como ondas "stop-and-go", efeito "mais rápido é mais lento" e "arqueamento e entupimento" $[2,24]$.

A natureza estocástica do modelo de simulação adotado prevê flutuações aleatórias que podem influir nos resultados das simulações. Em razão desse componente aleatório, que não pode ser controlado pelo pesquisador, foram realizadas 10 simulações de cada cenário analisado, adotando-se a média dos tempos máximos de evacuação em cada um deles.

4.

\section{RESULTADOS}

O Gráfico 1 apresenta os resultados obtidos para o primeiro conjunto de cenários, com um único corredor, especificamente para a velocidade desejada (Vd) de 2,5 m/s, que corresponde à velocidade de pessoas em pânico. O gráfico apresenta ainda os resultados obtidos por Dias et al. [4] nos experimentos realizados com 250 formigas impelidas a abandonarem as câmaras de ensaio após a introdução de um repelente. 
Gráfico 1 - Comparação entre fluxos de saída de formigas e humanos virtuais nas soluções com apenas 1 corredor (conjunto i)

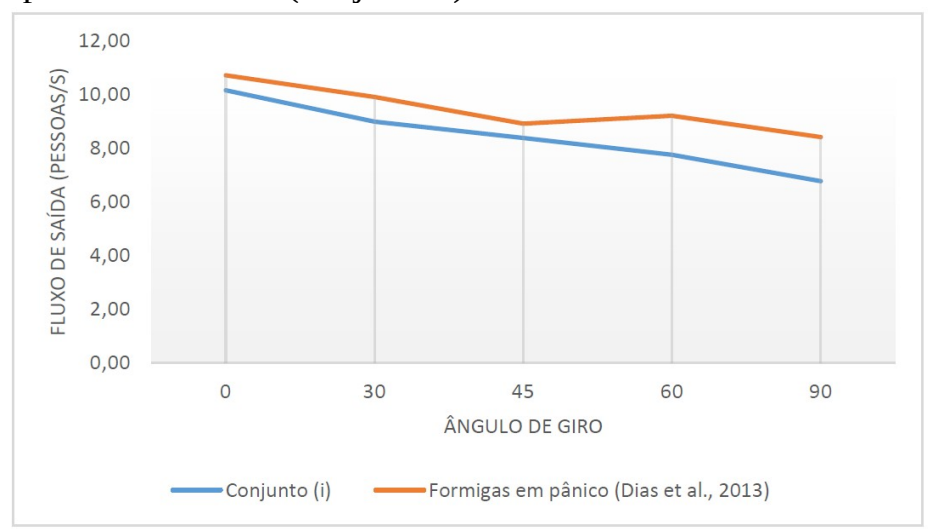

Fonte: Elaborado pelos autores.

Vê-se, no Gráfico 1, que os valores obtidos nas simulações computacionais estão muito próximos aos valores obtidos por Dias et al. [4] para formigas em pânico. Diferem os resultados obtidos para o ângulo de $60^{\circ}$, para o qual os autores sugerem que as formigas podem apresentar alguma facilidade em relação aos seus padrões morfológicos e de locomoção. A observação do Gráfico 1 permite inferir que, para a $\mathrm{Vd}=2,5 \mathrm{~m} / \mathrm{s}$, o aumento do ângulo de giro exigido pelo formato do corredor de saída provoca o aumento do tempo de escape. No entanto, observando-se o Gráfico 2, vê-se que o ângulo de $60^{\circ}$ foi mais eficaz que os demais (exceto o de $0^{\circ}$ ) para os conjuntos (ii) e (iii), ambos com dois corredores de saída. Destaca-se que o Gráfico 2 apresenta os resultados para $\mathrm{Vd}=2,5 \mathrm{~m} / \mathrm{s}$, ou seja, velocidade de pessoas em pânico.

Gráfico 2 - Fluxo de saída para os conjuntos (i), (ii) e (iii), sendo Vd = 2,5 m/s

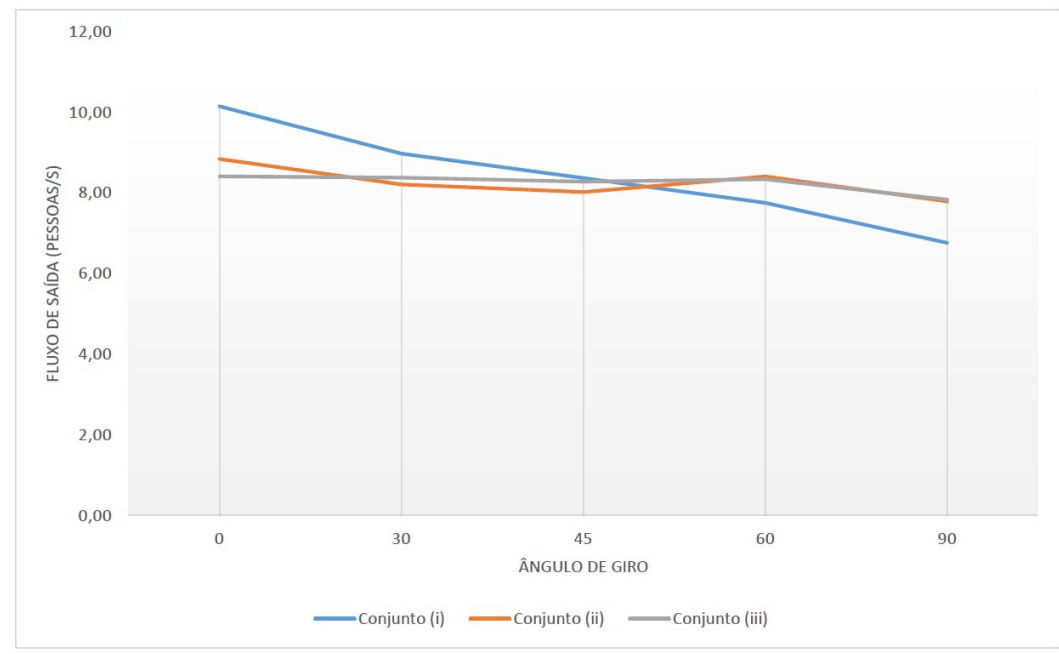

Fonte: Elaborado pelos autores.

O Gráfico 3 apresenta os resultados obtidos para a velocidade de 1,32 m/s, que corresponde à velocidade normal de caminhada dentro de uma multidão. Para esta velocidade, o conjunto (i), com um único corredor de saída, seguiu o mesmo padrão obtido para Vd = 2,5 $\mathrm{m} / \mathrm{s}$, ou seja, o aumento do ângulo de giro resultou em perda de eficácia no fluxo de saída. Para o conjunto (ii), com dois corredores de saída, o ângulo de $60^{\circ}$ apresentou a melhor eficácia, excetuando-se o corredor reto $\left(0^{\circ}\right)$, tal qual os resultados obtidos por Dias et al. [4]. 
E, para o conjunto (iii), também com dois corredores de saída, o ângulo de $45^{\circ}$ foi o que apresentou a melhor eficácia no fluxo de evacuação, que foi decaindo para ângulos maiores.

Gráfico 3 - Fluxo de saída para os conjuntos (i), (ii) e (iii), sendo Vd =1,32 m/s

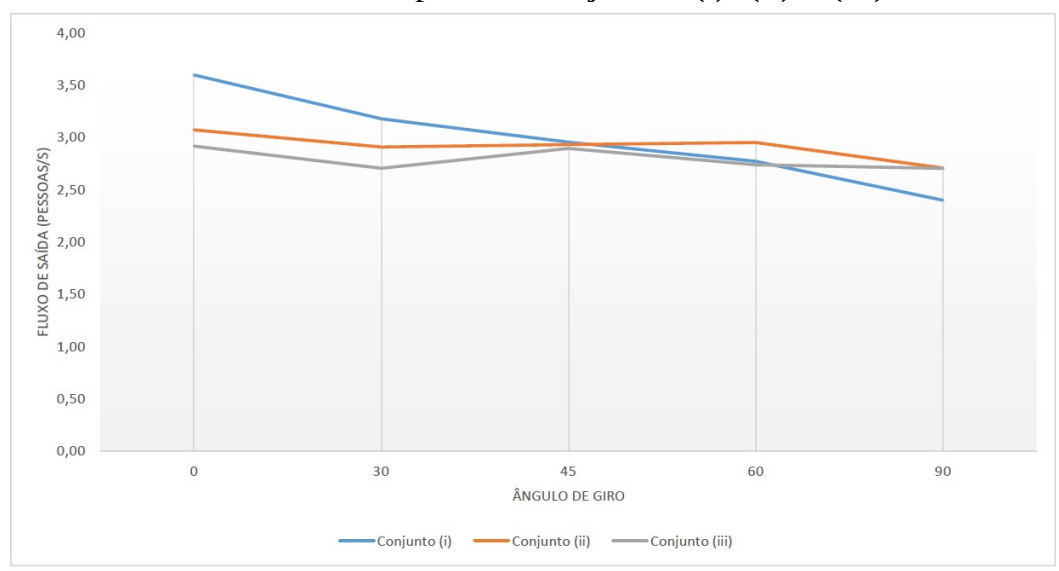

Fonte: Elaborado pelos autores.

As Figuras 3 a 5 buscam representar o que foi observado na análise qualitativa das simulações realizadas. Vê-se que, quanto maior a largura do corredor, menor o índice de aproveitamento da área de escape (área do corredor) pelos usuários. Fato que também pode ser observado, em menor proporção, com o aumento do ângulo de giro exigido pelo desenho do corredor.

Figura 3 - Captura de tela aos 8 segundos do início da simulação $(\mathrm{Vd}=2,5 \mathrm{~m} / \mathrm{s})$

$45^{\circ}$

(i)

(ii)

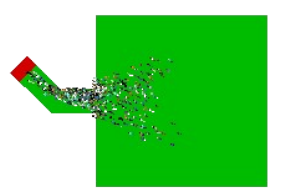

$60^{\circ}$

$90^{\circ}$
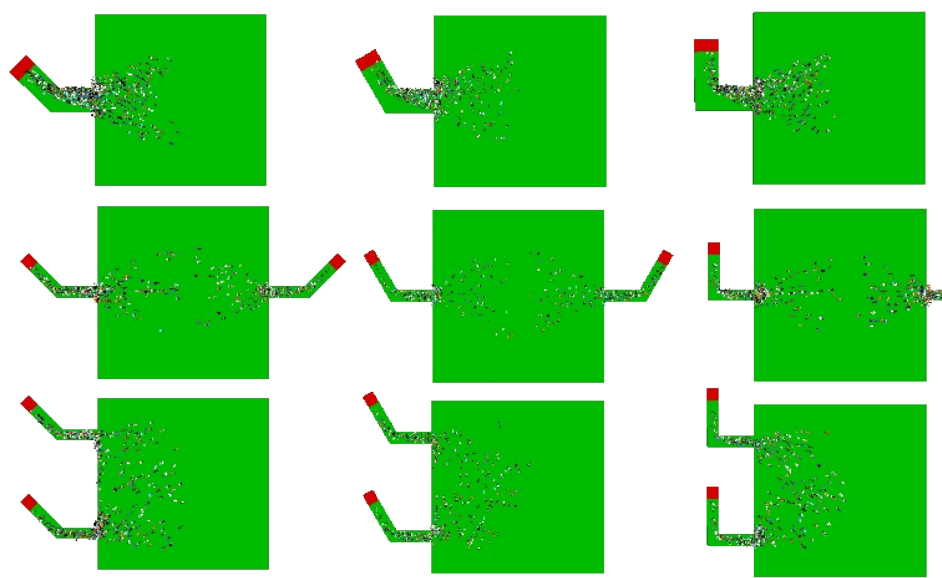

(iii)

Fonte: Elaborado pelos autores.
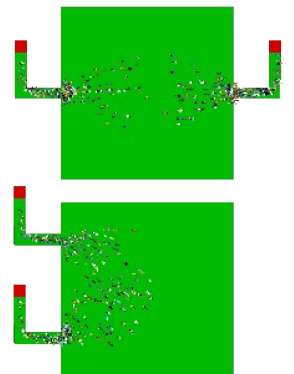
Figura 4 - Captura de tela aos 16 segundos do início da simulação $(\mathrm{Vd}=2,5 \mathrm{~m} / \mathrm{s}$ ) $45^{\circ}$

$60^{\circ}$

$90^{\circ}$

(i)
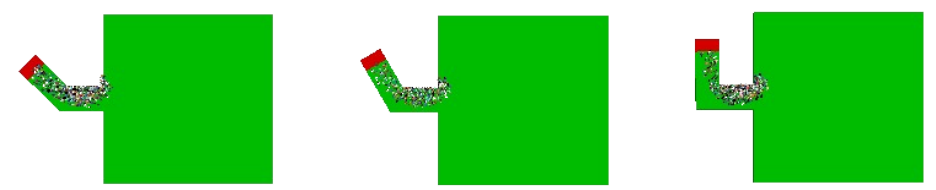

(ii)

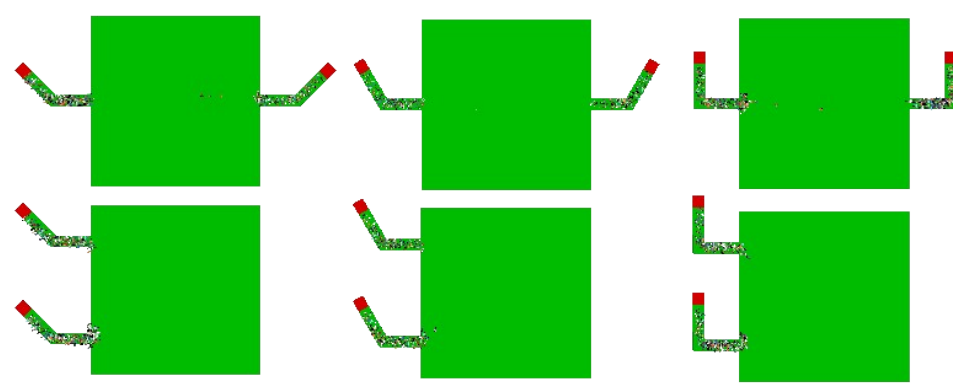

Fonte: Elaborada pelos autores.

Figura 5 - Captura de tela aos 24 segundos do início da simulação $(\mathrm{Vd}=2,5 \mathrm{~m} / \mathrm{s})$

$45^{\circ}$

(i)

(ii)

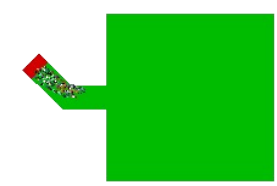

$60^{\circ}$

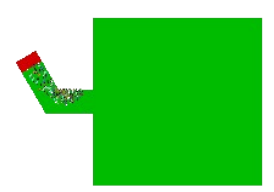
$90^{\circ}$

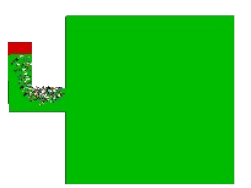

(iii)

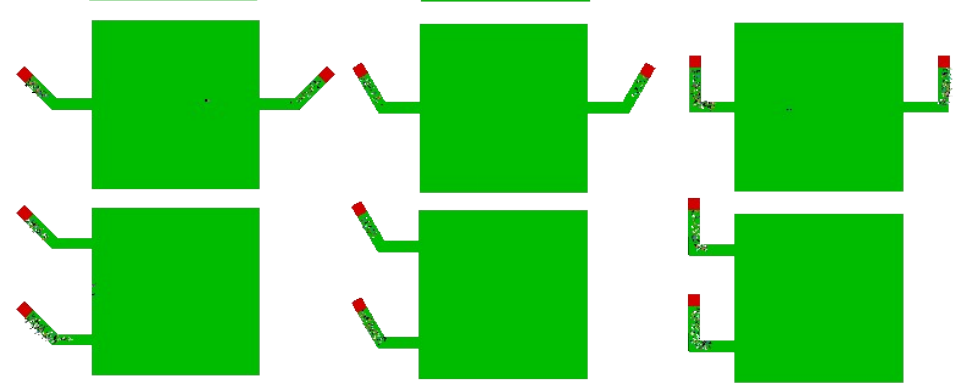

Fonte: Elaborada pelos autores.

A Tabela 1 apresenta a média dos tempos máximos de evacuação de cada um dos cenários analisados. Nela também é possível observar o percentual máximo de ganho de eficácia no tempo de evacuação em relação às alterações arquitetônicas propostas.

Tabela 1 - Média dos tempos máximos de evacuação

$$
\begin{array}{cccccc}
0^{\circ} & 30^{\circ} & 45^{\circ} & 60^{\circ} & 9^{\circ} & \begin{array}{c}
\text { Aumento da } \\
\text { eficácia da }
\end{array}
\end{array}
$$$$
\text { evacuação** }
$$

\begin{tabular}{lcccccc}
\hline conjunto (i) & 24,65 & 27,88 & 29,9 & 32,26 & 36,99 & $33,36 \%$ \\
\hline conjunto (ii) & 28,29 & 30,47 & 31,17 & 29,75 & 32,11 & $11,90 \%$ \\
\hline conjunto (iii) & 29,74 & 29,86 & 30,2 & 30,01 & 31,93 & $6,86 \%$ \\
\hline Aumento da & $17,11 \%$ & $8,50 \%$ & $4,07 \%$ & $7,78 \%$ & $13,68 \%$ &
\end{tabular}

eficácia da

evacuação*

* aumento da eficácia em relação à adoção de uma ou duas saídas e ao seu posicionamento.

** aumento da eficácia em relação ao ângulo adotado no projeto da saída.

Fonte: Elaborada pelos autores. 


\section{CONSIDERAÇÕES FINAIS}

Considerando a velocidade de pânico $(2,5 \mathrm{~m} / \mathrm{s})$, os resultados obtidos com o primeiro conjunto de cenários simulados mostraram-se muito semelhantes àqueles obtidos por Dias et al. [4] para formigas em pânico. Eles evidenciaram que o aumento do ângulo de giro repercute diretamente no fluxo de saída (pessoas/s). Ressalta-se que esse conjunto de cenários, com um único corredor de saída, seguiu o mesmo layout apresentado por Dias et al. [4], adequando-se as dimensões conforme proposto em Shiwakoti et al. [6].

Os cenários com dois corredores, por sua vez, quando comparados aos cenários com um único corredor, apresentaram-se mais eficazes para ângulos de giro de $60^{\circ}$ e $90^{\circ}$. Uma análise qualitativa das simulações sugere que giros de até $45^{\circ}$ não reduzem o aproveitamento, por parte dos usuários, da área de escape representada pelo corredor. Ângulos de giro maiores, como os de $60^{\circ}$ e $90^{\circ}$, ao induzirem os pedestres a se locomoverem na parte mais interna, promovem a sua subutilização. Conforme apontam os resultados encontrados para os conjuntos (ii) e (iii), essa capacidade pode ser melhor aproveitada com a adoção de dois corredores, mantendo-se a mesma largura total. Nas simulações realizadas para a velocidade de pânico, a adoção de dois corredores melhorou em até $15 \%$ o fluxo de saída para o corredor com ângulo de giro de $90^{\circ}$ e até $8 \%$ para o de $60^{\circ}$.

Quanto às simulações realizadas para a velocidade de $1,32 \mathrm{~m} / \mathrm{s}$, que corresponde à velocidade normal de caminhada dentro de uma multidão, os resultados obtidos apresentam tendências semelhantes aos da velocidade de pânico, com fluxo de escape médio cerca de $65 \%$ menor. No entanto, para o ângulo de $60^{\circ}$, o conjunto (iii), com dois corredores de saída posicionados em paredes opostas, apresentou eficácia menor que o conjunto (i), com um único corredor, nisso diferindo-se da velocidade de pânico.

De maneira geral, viu-se que os ganhos relacionados ao aumento da eficácia de uma evacuação podem chegar a 33,36\% quando considerados diferentes ângulos de giro e a $17,11 \%$ quando consideradas diferentes quantidades e posicionamento das saídas de emergência. Nos cenários simulados neste trabalho, esses percentuais representam, respectivamente, 12,34 segundos e 5,09 segundos. Importa destacar que esses valores correspondem à fuga por um corredor de 12,64 m e que, ao considerar-se percursos mais longos, potencializa-se a contribuição do projeto arquitetônico em reduzir o tempo de escape de uma edificação e, assim, salvaguardar vidas.

\section{Agradecimento}

O presente trabalho foi realizado com apoio da Coordenação de Aperfeiçoamento de Pessoal de Nível Superior - Brasil (CAPES) - Código de Financiamento 001 - 88887.091740/2014-01 - Projeto PROALERTAS.

6.

\section{REFERÊNCIAS BIBLIOGRÁFICAS}

[1] HAGHANI, M.; SARVI, M. Crowd behaviour and motion: empirical methods. Transportation Research Part B: Methodological, v. 107, p. 253-294, 2018. https://doi.org/10.1016/j.trb.2017.06.017.

[2] HELBING, D.; FARKAS I.; VICSEK, T. Simulating dynamical features of escape panic. Nature, v. 407, p. 487-490, 2000. 
[3] SHIWAKOTI, N.; SARVI, M.; ROSE, G.; BURD M. Enhancing the safety of pedestrians during emergency egress: can we learn from biological entities?

Transportation Research Part C: Emerging Technologies, v. 2137, p. 31-37, 2009. https://doi.org/10.3141/2137-04.

[4] DIAS, C.; SARVI, N.; EJTEMAI, O.; BURD, M. Investigating collective escape behaviours in complex situations. Safety Science, v 60, p. 87-94, 2013. https://doi.org/10.1016/j.ssci.2013.07.005.

[5] LEE, R. S. C.; HUGHES, R. L. Prediction of human crowd pressures. Accident analysis \& prevention, v. 38, n. 4, p. 712-722, 2006. https://doi.org/10.1016/j.aap.2006.01.001.

[6] SHIWAKOTI, N.; SARVI, M.; BURD, M. Using non-human biological entities to understand pedestrian crowd behaviour under emergency conditions. Safety Science, v. 66, p. 1-8, 2014. https://doi.org/10.1016/j.ssci.2014.01.010.

[7] TRAFFEX 2013 Show Preview. Traffex \& Parkex 16-18 April, NEC, Birmingham. Traffic Engineering \& Control, p. 75-81, apr. 2003.

[8] YANAGISAWA, D., NISHI, R.; TOMOEDA, A.; OHTSUKA, K.; KIMURA, A.; SUMA, Y.; NISHINARI, K. Study on efficiency of evacuation with an obstacle on hexagonal cell space. SICE Journal of Control, Measurement, and System Integration, v. 3, n. 6, p. 395-401, 2010. https://doi.org/10.9746/jcmsi.3.395.

[9] COURTINE, G.; SCHIEPPATI, M. Human walking along a curved path. I. Body trajectory, segment orientation and the effect of vision. European Journal of Neuroscience, v. 18, n. 1, p. 177-190, 2003.

[10] MOUSSAÏD, M.; HELBING, D.; THERAULAZ, G. How simple rules determine pedestrian behaviour and crowd disasters. Proceedings of the National Academy of Science of the United States of America, v. 108, n. 17, p. 6884-6888, 2011.

[11] HELBING, D.; MUKERJI, P. Crowd disasters as systemic failures: analysis of the love parade disaster. EPJ Data Science, v. 1, n. 1, p. 7, 2012. https://doi.org/10.1140/epjds7.

[12] STEFFEN, B.; SEYFRIED, A. Modelling of pedestrian movement around 90 and 180 bends. In: The First International Conference on Soft Computing Technology in Civil, Structural and Environmental Engineering. Civil-Comp Press, Stirlingshire, UK. 2009.

[13] ALTSHULER, E.; RAMOS, O.; NÚÑEZ, Y.; FERNÁNDEZ, J.; BATISTA-LEYVA, A. J.; NODA C. Symmetry breaking in escaping ants. The American Naturalist, v. 166 n. 6, p. 643-649, 2005.

[14] SHIWAKOTI, N.; SARVI, M.; ROSE, G.; BURD, M. Biologically inspired modelling approach for collective pedestrian dynamics under emergency conditions. Transportation Research Record, v. 2196, p. 176-184, 2010.

[15] NISHINARI, K.; SUGAWARA, K.; KAZAMA, T.; SCHADSCHNEIDER, A.; CHOWDHURY, D. Modelling of self-driven particles: foraging ants and pedestrians. Physica A, v. 372, n. 1, p. 132-141, 2006. https://doi.org/10.1016/j.physa.2006.05.016.

[16] SORIA, S. A.; JOSENS, R.; PARISI D. R. Experimental evidence of the "Faster is Slower” effect in the evacuation of ants. Safety Science, v. 50, n. 7, p. 1584-1588, 2012. https://doi.org/10.1016/j.ssci.2012.03.010.

[17] PETERS H. R. The Ecological Implications of Body Size. Victoria: Cambridge University Press, 1999. 
[18] HELBING, D.; BUZNA, L.; JOHANSSON, A.; WERNER, T. Self-Organized Pedestrian Crowd Dynamics: Experiments, Simulations, and Design Solutions. Transportation Science, v. 39, n. 1, p. 1-24, 2005.

[19] HELBING, D.; FARKAS, I.; MOLNÁR, P.; VICSEK, T. Simulation of Pedestrian Crowds in Normal and Evacuation Situations. In: Schreckenberg, M.; Sharma, S. (eds). Pedestrian and Evacuation Dynamics. Springer, Berlin, p. 21-58, 2002.

[20] YU, W.; CHEN, R.; DONG, L.; DAI, S. Centrifugal force model for pedestrian dynamics. Physical Review E, v. 72, n. 026112, p. 1-7, 2005. https://doi.org/10.1103/PhysRevE.72.026112.

[21] FRANK, G. A.; DORSO, C. O. Room evacuation in the presence of an obstacle. Physica A: Statistical Mechanics and its Applications (Amsterdam), v. 390, n. 11, p. 213-2145, 2011. https://doi.org/10.1016/j.physa.2011.01.015.

[22] SHIWAKOTI, N.; SARVI, M. Enhancing the panic escape of crowd through architectural design. Transportation Research Part C: Emerging Technologies, v. 37, p. 260-267, 2013. https://doi.org/10.1016/j.trc.2013.04.009

[23] JIANG, L.; LI, J.; SHEN, C.; YANG, S.; HAN, Z. Obstacle Optimization for Panic Flow Reducing the Tangential Momentum Increases the Escape Speed. PLoS ONE, v. 9, n. 12, p. e115463, 2014. https://doi.org/10.1371/journal.pone.0115463.

[24] JOHANSSON, A.; HELBING, D.; SHUKLA, P. K. Specification of the social force pedestrian model by evolutionary adjustment to video tracking data. Advances in Complex Systems, v. 10, n. supp02, p. 271-288, 2007. https://doi.org/10.1142/S0219525907001355. 